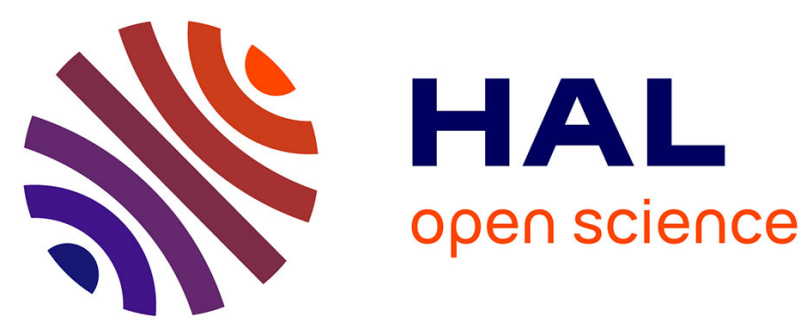

\title{
Thermomechanical characterization of stress localization in glass: An experimental and numerical study
}

\author{
Guillaume Corvec, Eric Robin, Jean-Benoit Le Cam, Pierre Lucas,
} Jean-Christophe Sangleboeuf, Frederic Canevet

\section{- To cite this version:}

Guillaume Corvec, Eric Robin, Jean-Benoit Le Cam, Pierre Lucas, Jean-Christophe Sangleboeuf, et al.. Thermomechanical characterization of stress localization in glass: An experimental and numerical study. Strain, 2017, 53 (4), pp.e12234. 10.1111/str.12234. hal-01578403

HAL Id: hal-01578403

https://hal-univ-rennes1.archives-ouvertes.fr/hal-01578403

Submitted on 23 Sep 2020

HAL is a multi-disciplinary open access archive for the deposit and dissemination of scientific research documents, whether they are published or not. The documents may come from teaching and research institutions in France or abroad, or from public or private research centers.
L'archive ouverte pluridisciplinaire HAL, est destinée au dépôt et à la diffusion de documents scientifiques de niveau recherche, publiés ou non, émanant des établissements d'enseignement et de recherche français ou étrangers, des laboratoires publics ou privés. 


\title{
Thermomechanical characterization of stress localization in glass: an experimental and numerical study
}

\author{
Guillaume Corvec ${ }^{1}$, Eric Robin ${ }^{1}$, Jean-Benoît Le Cam ${ }^{1, \dagger}$, Pierre Lucas ${ }^{2}$, Jean-Christophe \\ Sangleboeuf ${ }^{1}$ and Frederic Canevet ${ }^{3}$ \\ ${ }^{1}$ Université de Rennes 1, Institut de Physique UMR 6251 CNRS/Université de Rennes 1, Campus \\ de Beaulieu, Bât. 10B, 35042 Rennes Cedex, France. \\ ${ }^{2}$ Arizona Materials Laboratory, 4715 East Fort Lowell Rd, Tucson, AZ 85712, USA \\ ${ }^{3}$ Cooper Standard France, 194 route de Lorient, 35043 Rennes - France.
}

Summary. Thermoelastic stress analysis (TSA) and quantitative calorimetry are full-field non-contact techniques widely used to study the thermo-mechanical behavior of materials. The first one linearly relates the sum of the principal stresses to the temperature variation and the second one can be used to measure the mechanical dissipation. However, brittle materials such as glass are a priori bad candidates for these techniques. Indeed, their low temperature variations under loading lead to very noisy infrared images and their brittle mechanical behavior does not allow to deform them significantly. In the present paper, the thermo-mechanical characterization of a holed glass sample under cyclic loading is performed. A preliminary new filtering methodology has been applied to the thermal movie to remove the noise. The stress field obtained from the TSA is well correlated to the finite element model showing that this technique is adapted to study the thermoelastic response of brittle materials. Finally, the corresponding calorimetric response has been determined by using a simplified formulation of the heat diffusion equation. This permits to quantify heat sources and to carry out energy balances.

Keywords: Infrared thermography; denoising methodology; inorganic oxide glass; Thermoelastic Stress Analysis; quantitative calorimetry

\section{Introduction}

Thermoelastic and calorimetric effects accompanying the deformation of materials have been widely studied in the literature. They provide information of importance to better understand and model the deformation processes. Two main techniques are generally used: the so-called Thermoelastic Stress Analysis (TSA) $(1 ; 2)$ and the quantitative calorimetry $(3 ; 4 ; 5)$. These non-contact techniques are based on thermal measurement at the surface of the material under study. Since the 1980's, they have experienced an impressive expansion. TSA technique permits to determine the stress field at the surface of materials (6), to measure residual stresses (7), the stress intensity factor at crack tips (8) and, coupled with photoelasticity, to identify the principal stresses (9). Quantitative calorimetry allows the study of several phenomena such as Lüders or Portevin-Le Chatelier plastic instabilities (10), rubber elasticity (11) or mechanical fatigue $(12 ; 13)$. Most materials have already benefited from these techniques including smart memory alloys (14), aluminum alloys (10), polymers (15), composites $(16 ; 17 ; 18 ; 19 ; 20)$ and elastomers $(11 ; 21)$. These materials exhibit temperature variations in the range of one degree or more. In these conditions the experimental noise does not extensively affect the measurement and basic filters can be used to detect and to quantify temperature variations.

Concerning inorganic glass materials, studies generally focus on crack tip movement (22), mechanical properties $(23)$ or fracture $(24 ; 25)$, but rarely on their thermo-mechanical properties. Although 
glasses are used in a wide range of applications due to their transparency, heat resistance, pressure resistance, and chemical resistance, their fragility and low fracture toughness prevent them from use in most mechanical components. While their mechanical tensile strength is theoretically close to $35 \mathrm{GPa}$, experimentally measured values are rather close to several tens or hundreds of MPa. This is explained by the fact that the limit to failure of glasses is driven by their surface defects. Therefore, the strain level applied for a mechanical test must be very low, approximately $10^{-5}$. In addition, since their thermal conductivity is low, temperature variations accompanying deformation of glasses are very low too. Hence, to the best of our knowledge, only two studies have been dedicated to the thermal and thermo-mechanical response of inorganic glasses $(26 ; 27)$. These studies have shown that it was possible to detect very low temperature variations (in the range of few tenths of a degree). The temperature variations amplitude was satisfactorily determined but only in the case of moderate thermal gradient. In the present study, we use a filtering methodology adapted to measure strong thermal gradients even in the case of low temperature variations while preserving the spatial resolution equal to 1 pixel. This enables us to characterize the thermal and calorimetric effects of stress and strain concentrations.

The experimental set up devised to induce a strong stress concentration in a disc of glass under compressive loading is described in section 2 along with the full thermal and kinematic measurements. Sections 3 briefly describes the filtering method and recalls the framework of TSA and quantitative calorimetry. The results of the thermal field measurements at the specimen surface is then presented in section 4 and the stress field obtained from the temperature measurements is discussed and compared to the results of a numerical simulation carried out by using the finite element method. Lastly in this section, the heat sources produced or absorbed at the surface of the glass specimen are presented and the methodology for identifying mechanical dissipation is given. Even though no mechanical dissipation is expected at ambient temperature in soda lime glass, this methodology has to be validated before being applied at higher temperature, i.e. at temperature close to glassy transition where the behavior under multi-axial loading is not well-known.

\section{Experimental set-up}

\subsection{Material and specimen geometry}

The material considered here is a soda lime glass. The specimen geometry is depicted in Figure 1. It corresponds to a disc of $2.1 \mathrm{~mm}$ in thickness and $29.7 \mathrm{~mm}$ in diameter with three elliptical holes. Other dimensions are given in the figure. Holes were cut with a water jet cutting machine.

\subsection{Loading conditions}

The disc was subjected to cyclic compressive loading by means of a 5543 Instron testing machine. An overview of the experimental setup is given in Figure 2. The test was conducted under a prescribed periodic triangular signal. The minimum and maximum values of the compression force are $5 \mathrm{~N}$ and $520 \mathrm{~N}$, respectively. It should be noted that the compression force never comes back to zero in order to keep the contact between the moving grip and the disc, which avoids any problem in the testing machine control. The sample was submitted to ten cycles at a frequency of $2.9 \mathrm{~Hz}$.

\subsection{Infrared measurement}

Temperature measurements were performed at ambient temperature using a X6540sc FLIR infrared camera. It features a focal plane array of $640 \times 512$ pixels and detectors with a wavelength in the range of 1.5-5.1 $\mu \mathrm{m}$. The integration time was equal to $1000 \mu \mathrm{s}$ and the acquisition frequency was set at $100 \mathrm{fps}$. The resolution of the thermal measurement is equal to $20 \mathrm{mK}$ at $25^{\circ} \mathrm{C}$. The thermal emissivity of the material is close to 1 ( $>0.84$ for soda lime glass). The spatial resolution of the thermal measurement was such that the size of the pixel was equal to $63 \mu \mathrm{m}$. This resolution was obtained by adding a converter (ring extension). It enables us to increase the spatial resolution by a factor between 2.7 and 4.76. A black body-like box, which is not shown in Fig. 2, surrounds the 
specimen and a part of the grips in order to stabilize the external radiations during the mechanical test. In order to ensure that the internal temperature of the camera was stabilized before performing the measurements, it was switched on for 2 hours before the experiment. Then, the Non Uniformity Correction (NUC) of the matrix of detectors was performed by using the manufacturer's protocole. Once the temperature field at the specimen surface was stabilized, the temperature measurement was started. Then, the mechanical loading was applied.

\section{Background}

\subsection{Data processing and filtering}

At ambient temperature, glassy materials are brittle and do not undergo high load levels without breaking. Moreover, their thermal expansion coefficients are low. Consequently, the thermal activity of soda lime glass during a mechanical test is also low and the thermal signal is noisy. Processing thermal images therefore requires to filter the thermal data. In previous studies, we have solved this problem by performing cyclic tests and by using a spatio- temporal filter (26). This approach provides satisfactory results in case of moderate spatial gradients, such as those obtained in case of the three point bending tests. However, this approach smoothes the signal at each pixel and spatial gradients cannot be characterized anymore in case of higher spatial gradients. To address such issue, we have recently developed a new denoising methodology that enables us to keep the spatial resolution equal to 1 pixel (28). However, this method requires tracking each material point to measure its temperature. This may become problematic if large deformation occurs during image collection under mechanical loading. In the case of inorganic glasses the deformation during the mechanical test is low. So the temperature measurements can be processed by assuming that they are not affected by the displacements of the points observed by the infrared camera. To validate this assumption, a white mark was painted on the sample surface, close to the moving grip, i.e. in a zone where the displacement is assumed to be the highest, and followed during the test by using a mark tracking method described in further details in (29). The kinematic camera used for that process was an EDMUND OPTICS EO-0413M camera with a resolution of 752 × 480 pixels and a frame rate of $50 \mathrm{fps}$. The spatial resolution was equal to $85 \mu \mathrm{m}$. Figure 3 presents the displacement in $m m$ obtained along the $x$ (denoted $\mathrm{X}$ ) and $y$ (denoted $\mathrm{Y}$ ) axes during the cyclic mechanical loading, once the preloading is applied ( $5 \mathrm{~N}$ in compressive loading, see paragraph 2.2). It is observed that the shape of the displacement signal is not exactly triangular. As the material elasticity is linear and temperature variations are proportional to stress variations, temperature variation and displacement evolve in the same way. As displacement measurement is less noisy than temperature variation measurement, the characteristics of the filter for temperature variation denoising are defined from the spectral analysis of the displacement signal. Figure 4 shows the maximum displacement from the point of origin along the $x$ and $y$ axis during the full mechanical loading cycle. This diagram show that the maximum displacement reached along the $x$ and $y$ axes is inferior to $0.010 \mathrm{~mm}$ and $0.04 \mathrm{~mm}$ respectively. The displacement of the white mark is therefore included within a window inferior to $0.040 \times 0.010 \mathrm{~mm}$ square. Since the spatial resolution of the temperature measurement is equal to $0.063 \mathrm{~mm}$, it is therefore not significantly affected by the mechanical displacement and compensating the displacement of the points is not required. The denoising methodology is divided in two steps. The first one consists in removing the noise from the temporal signal at each pixel by applying a purely temporal filter. The denoised temperature variation is obtained by selecting the background and the first two harmonics given by the STFFT of the displacement signal. The harmonic number has been optimized from the minimization of a cost function defined as the quadratic difference between the denoised signal and the displacement measurement.

Figure 5 presents a temperature evolution before and after filtering at point $A$. This allows us to reconstruct the temperature variation at this pixel without noise. But while this step effectively reduces the temporal signal noise at each pixel, the infrared images may still appear noisy. This is due to a random distribution of the residual offset value of each (independent) pixel. Hence in the second step, the residual offset is identified by considering thermal images before applying the mechanical loading. In this case, the temperature variation field is homogeneous and the value of temperature 
variation at each pixel is theoretically equal to zero. Therefore, a residual offset can be identified and removed from each pixel of the infrared camera. Further details are provided in (28). This approach was used in the present study to denoise thermal measurements.

\subsection{Thermoelastic stress analysis procedure}

The TSA approach permits to link temperature and stress variations. Stress variation corresponds to change in the sum of principal Cauchy stresses $(\operatorname{tr}(\sigma))$, which corresponds to three times the hydrostatic stress. The thermomechanical framework of TSA is described following the formalism introduced in (30). The thermodynamic process is considered as a quasi-static phenomenon. The state of any material volume element is defined by $N$ state variables: temperature $T$, one of the strain tensors denoted $E$ and some internal variables $V_{1}, V_{2}, \ldots, V_{N-2}$ such as plastic strain or volume fractions of some phases. The specific free energy potential is denoted $\Psi\left(T, E, V_{k}\right)$. Considering the first and second principles of thermodynamics and assuming that the Fourier's law is used to model the heat conduction, the heat diffusion equation can be written as follows:

$$
\rho C_{E, V_{k}} \dot{T}-\operatorname{div}(K \operatorname{grad} T)-r_{e x t}=\underbrace{d_{1}+\rho T \frac{\partial^{2} \Psi}{\partial T \partial E} \dot{E}+\rho T \frac{\partial^{2} \Psi}{\partial T \partial V_{k}} \dot{V}_{k}}_{s}
$$

where $\rho$ is the density, $C_{E, V_{k}}$ is the specific heat at constant $E$ and $V_{k}, K$ is the thermal conductivity tensor and $r_{e x t}$ is the external heat source. The right-hand side of equation (1) represents the heat sources $s$ produced by the material itself. It can be split in different terms:

- the intrinsic dissipation $d_{1}$ (or mechanical dissipation, to be distinguished from the thermal dissipation in the Clausius-Duhem inequality). This positive quantity corresponds to the heat production due to some mechanical irreversibilities such as internal friction or damage;

- the thermomechanical couplings $\left(s-d_{1}\right)$ : they correspond to the couplings between the temperature and the other state variables. The coupling between temperature and strain $\rho T \frac{\partial^{2} \Psi}{\partial T \partial E} \dot{E}$ is the thermoelastic coupling $(12 ; 13)$.

Assuming that heat conduction is isotropic, heat diffusion equation is written as:

$$
\rho C_{E, V_{k}} \dot{T}-k \Delta T-r_{e x t}=s
$$

where $\Delta$ is the laplacian operator and $k$ the thermal conductivity. For a temperature changes $\theta=$ $T-T_{r e f}$, defined with respect to a reference temperature field $T_{r e f}$, itself defined when no heat source is produced by the specimen, and by considering that the external heat source $r_{e x t}$, the density and the heat capacity are constant over the duration of the test, equation 2 becomes:

$$
\rho C \frac{\partial \theta}{\partial t}-k \Delta \theta=s
$$

For a thermoelastic behavior, the heat source $s$ produced by the material is composed of the thermoelastic (isentropic) coupling term and possibly mechanical (intrinsic) dissipation $d_{1}$. It is defined as a non-negative quantity. For glass materials, which are assumed to behave as isotropic and thermoelastic materials, the thermoelastic coupling term is given by $-\alpha T_{0} \frac{\partial \operatorname{tr}(\sigma)}{\partial t}$, where $\alpha$ is the coefficient of thermal expansion and $T_{0}$ the mean temperature of the specimen over the test (in Kelvin). Thus, we have:

$$
\rho C \frac{\partial \theta}{\partial t}-k \Delta \theta=-\alpha T_{0} \frac{\partial t r(\sigma)}{\partial t}+d_{1}
$$

Equation 4 shows that, in the general case, cyclic mechanical loading leads to a temperature variation composed of a global increase (due to mechanical dissipation $d_{1}$ ) and to an oscillation at the same frequency as the load but out of phase (due to thermoelastic coupling). As mechanical dissipation due to fatigue can be considered as negligible over one cycle, the range of temperature oscillation 
$\Delta \theta$ during one cycle is directly proportional to the range of the sum of principal Cauchy stresses $\Delta \operatorname{tr}(\sigma)$ :

$$
\Delta \theta=-A \Delta \operatorname{tr}(\sigma)
$$

where $A$ is the so-called thermoelastic coefficient and is equal to $\frac{\alpha T_{0}}{\rho C}$. This gives rise to numerous applications in the field of thermoelastic stress analysis. The reader can refer to $(31 ; 2)$ for further information. For the material considered in this study, parameter $A$ is equal to $1.34110^{-3} \mathrm{~K} / \mathrm{MPa}$ at $25^{\circ} \mathrm{C}$.

\subsection{Heat source field reconstruction at the specimen surface}

A flat specimen is described within a $(x, y, z)$ cartesian coordinate system such that the $x$ and $y$ axes give the direction of the specimen plane and the $z$-direction is perpendicular to this plane. It should be noted that the maximum thickness must be weighed against the thermal conductivity of the material to ensure that temperature is homogeneous through the thickness. On the other hand, the temperature gradient $\partial T / \partial z$ is not constant near the front and back sides of the specimen due to heat exchanges with ambient air (32). Averaging Equation (2) through the thickness of the specimen leads to:

$$
\rho C_{E, V_{k}} \dot{\bar{T}}-k\left(\frac{\partial^{2} \bar{T}}{\partial x^{2}}+\frac{\partial^{2} \bar{T}}{\partial y^{2}}\right)-\frac{1}{e} k\left[\frac{\partial T}{\partial z}\right]_{-e / 2}^{e / 2}-r_{e x t}=s
$$

where $e$ is the specimen thickness and $\bar{T}$ is the mean through-thickness temperature. The material parameters $\rho, C_{E, V_{k}}, k$, the external heat sources $r_{\text {ext }}$ and the heat sources $s$ produced by the material itself are also assumed to be constant through the thickness of the specimen. By using temperature variation $\theta$ instead of temperature and by considering that the external heat sources $r_{e x t}$ and the material parameters $\rho, C_{E, V_{k}} k$ are constant during the test, the 2D-version of the heat diffusion equation is given by:

$$
\rho C_{E, V_{k}}\left(\dot{\theta}+\frac{\theta+T_{0}-T_{\mathrm{amb}}}{\tau}\right)-k \Delta_{2 D} \theta=s
$$

where $\Delta_{2 D}$ is the two-dimensional Laplace operator in the $(x, y)$ plane and $\tau$ is the time constant, which characterizes the heat exchange with ambient air. This time constant, which was identified from a return to ambient temperature, is equal to $114 \mathrm{~s}$. It should be noted that if the loading frequency is sufficiently high, adiabatic conditions are achieved over one cycle, and heat diffusion due to both the convection and the conduction can be neglected. The low thermal diffusivity $D$ of the soda lime glass and the frequency applied leads to a thermal diffusion length $\sqrt{\frac{D}{\pi f_{L}}}$ equal to $0.234 \mathrm{~mm}$ (33), which can be assumed lower than the spatial variations of stress in the disc as will be discussed below. Besides, considering the spatial resolution of the thermal measurement $(0.063 \mathrm{~mm})$, the heat conduction is detectable in a window of maximum 4 pixels only. We therefore consider that heat conduction does not significantly affect the temperature measurement. Finally, if ambient temperature remains constant over the test, the heat diffusion equation is given by:

$$
\rho C_{E, V_{k}}\left(\dot{\theta}+\frac{\theta}{\tau}\right)=s
$$

To estimate heat sources $s$ produced by the material, the procedure consists in calculating the left-hand side of Equation 8 by processing the temperature variation fields.

\section{Results and discussion}

\subsection{Temperature variation and stress variation fields}

Figure 6(a) presents the temperature variation map issued from denoised thermal field measurements. It should be noted that the maximum and minimum values of the temperature variation in this figure are not located in the contact zones, but at the disc borders, due to optical effects. Consequently, this anamorphosed image does permit to properly distinguish the distribution of the temperature variation 
due to the mechanical loading. In Figure 6(b), the thermal image scale is reduced in order to better highlight gradients in the temperature variation field. In this figure, each elliptical hole exhibits both positive and negative temperature variations. In order to quantitatively analyze these variations, four points have been chosen in different zones: points $A, B$ and $C$ are located in zones of high thermal activity (in the contact zone and near an elliptical hole); point $D$ is located at the specimen centre. Figures 7(a) and (b) show the temperature variation at these four points during the test. Several observations can be made from these figures:

- Point A exhibits the highest amplitude of temperature variation (approximately $0.23^{\circ} \mathrm{C}$ ). This is due to the high stress concentration induced by the contact process.

- Point $D$, which is equidistant from the elliptical holes, exhibits the lowest amplitude of temperature variation $\left(<0.025^{\circ} \mathrm{C}\right)$.

- At points $\mathrm{B}$ and $\mathrm{C}$, located near an elliptical hole, temperature variation evolves in opposition of phase with similar amplitudes (approximately $0.05^{\circ} \mathrm{C}$ ). However, further analysis of temperature maps indicate that the zone affected by spatial gradient of temperature variation is larger at point $\mathrm{B}$ than $\mathrm{C}$.

- The fact that the minimum value of the temperature variation is not zero at points $B$ and $C$ and that it is decreasing at point $A$ highlights the effect of heat diffusion by convection. This diffusion process is low, else the mean temperature variation would be rapidly equal to zero (see the high value of the time constant $\tau$ with respect to the signal period). This proves that the test is performed under quasi-adiabatic loading condition. This is an important finding, which validates the link between amplitude of temperature variation and amplitude of the sum of principal stresses.

To determine the stress field from the temperature variation $(\Delta \theta)$ field, a short-time fast Fourier transform was used. The change of the sum of principal stresses was then calculated with the help of Equation 5 applied at each pixel. It is signed by using the following equation:

$$
\Delta \operatorname{tr}(\sigma)_{\text {signed }}=\Delta \operatorname{tr}(\sigma) \times\left(-\frac{|\theta|}{\theta}\right) .
$$

Figure 8(a) gives the map of the signed $\Delta \operatorname{tr}(\sigma)$. Similarly to Figure 6(b), the scale has been fixed manually to better highlight the stress gradients. The figure shows that the elliptical holes strongly concentrate the stress. To better highlight the stress concentration due to contact, Figure 8(b) shows the stress map in the boxed zone in Figure 8(a). This boxed zone corresponds to a part of the contact zone with the moving grip. The maximum stress level reached $\left(\Delta \operatorname{tr}(\sigma)_{\text {signed }}>200 \mathrm{MPa}\right)$ is the maximum level in this part of the contact zone.

\subsection{FE analysis}

A finite element model of the holed disc has been performed with Abaqus software. Figure 9 presents the solid mesh of the disc. 38277 finite elements C3D8R (52084 nodes) have been used. The thickness of the disc has been discretized with 3 finite elements. Two circular analytical surfaces have been generated for applying the compressive load. They were considered as master surfaces for the contact configuration. Slave surfaces are located at the edges of the disc. The friction coefficient was set at 0.1 . For the calculation, we assume that the sliding is small between master and slave surfaces. In this case, each node of the slave surfaces does not interact with more than one finite element belonging to the master surfaces. This assumption is realistic and enables us to reduce the calculation time. The elastic parameters, Young modulus and Poisson coefficient, were set at $70 \mathrm{GPa}$ and 0.2 , respectively. A $520 \mathrm{~N}$ compressive loading was applied on the disc by the circular analytical surfaces.

The hydrostatic pressure field obtained is presented and compared to the experimental result in Figure 10. To better highlight the spatial gradients in the vicinity of the elliptical holes, the scale of the two maps has been set at $[-6.33,13.3] \mathrm{MPa}$. A very good correlation of the location of spatial gradient zone is found between numerical and experimental results. This is further highlighted in Figure 11, 
which shows the magnified region around the elliptical hole. The location and size of the stress gradient zones around the contact zone and close to the elliptical holes shows an excellent match between simulation and experiment. These results also highlighted that in zone of strong stress concentration, the fact that heat conduction takes place over 4 pixels (cf. section 3.3) does not significantly affect the value of the temperature.

In order to provide a more quantitative comparison of the stress distribution derived from simulation and experiment, Figure 12 presents the value of the hydrostatic pressure along a section between the contact zones (shown as dark and blue lines). Again, a very good correlation is obtained, especially for the lowest stress levels that induce temperature variation inferior to a tenth of a degree. The few mismatches can be related to optical effects such as the observation angle, the variation in the apparent emissivity, the variations in the external radiations during the test, but also to the contact conditions and to the value of the thermoelastic parameter. Other source of error could originate from the choice of the constitutive parameters and the contact parameters in the numerical simulation.

In conclusion, a very good correlation is found between the stress fields derived experimentally from the thermal activity and that derived from numerical simulations based on the known mechanical behavior of the sample. This excellent quantitative match therefore validate the experimental approach, which will be used in the future to characterize the non-predictable mechanical state at the surface of glasses under complex loading conditions, especially for temperature close to the glass transition.

\subsection{Heat source field reconstruction}

The heat source field has been reconstructed from temperature variation field and the simplified heat diffusion equation (see Eq. 8). Since pixels are independent and heat conduction is negligible, the spatial resolution of heat source is 1 pixel. Nevertheless, the temporal derivation of temperature variation affects the noise level and the resolution of the heat sources. Figure 13 presents the heat source map obtained for image 285. As expected, the heat source gradients can be deduced from the temperature variation map of Fig. 6(a), the value of $\frac{\theta}{\tau}$ being low compared to $\dot{\theta}$. Two zones are magnified in order to clearly illustrate the location and size of heat source gradients. The zone under the contact area shows the the maximum of heat sources which is $3^{\circ} \mathrm{C} / \mathrm{s}$. Concerning the zone around the elliptical hole, color scale has been reduced between -0.5 and $0.5^{\circ} \mathrm{C} / \mathrm{s}$ because edge effects artificially increase the extremum of the heat sources. Again, the zones in opposition of phase around the hole are clearly highlighted. Figures 14(a) and (b) show the heat source variation at points $A, B, C$ and $D$. For each of them, heat source oscillates around zero, meaning that no mechanical dissipation occurs. This is consistent with the fact that glass behaves as thermoelastic material at ambient temperature. The maximum value of the heat source is obtained at point $A$ $\left(>1.5^{\circ} \mathrm{C} / \mathrm{s}\right)$, located in the contact zone. Point $\mathrm{D}$, located at the disc center, far from the geometrical singularities induced by elliptical holes, exhibits very low heat source variation $\left(<0.25^{\circ} \mathrm{C} / \mathrm{s}\right)$. In the vicinity of one elliptical hole, at point $B$ and $C$, the heat sources oscillate in phase but with opposite signs. Results obtained in terms of heat source variation enables us to calculate the mechanical dissipation by subtracting the heat absorbed to the heat produced over one mechanical cycle. An average over several cycles can be done to increase the resolution of the calculation. Obviously in the present case, the mechanical dissipation was found to be nul, but the final aim is to apply this methodology to characterize the occurrence of mechanical dissipation when the ambient temperature is increased.

\section{Conclusion}

Thermomechanical characterization of a brittle material is performed in the case of thermal signals of low amplitude and stress concentrations due to geometrical singularities. A disc of glass containing three elliptical holes has been submitted to a cyclic compressive load to investigate stress and strain concentration. Full thermal field measurement has been performed by using infrared thermography 
during cyclic loading. The temperature variation images stored during the test were denoised by using a methodology that permits to keep the spatial resolution equal to 1 . The framework of the TSA was applied to map the stress field at the surface of the specimen. Experimental results were quantitatively compared with numerical simulation issued from a finite element analysis, which serves as validation of the experimental methodology to process stresses from temperature measurements. Finally, the calorific response of the material has been determined from the heat diffusion equation and the temperature variation field. Its spatial resolution was also equal to 1 pixel. This method should permit to identify mechanical dissipation in case of complex loading conditions, i.e. for which no prediction is possible, as those encountered when the temperature is increased close to the glass transition temperature and the mechanical field is heterogeneous.

\section{Acknowledgements}

This work has received the financial support of AIS Scientific Grant from Rennes Métropole (2012), Mission of Resources and Skills Technology (MRCT) Grant from French National Center for Scientific Research (2012), Mission for Interdisciplinary (MI) Grant from French National Center for Scientific Research (2013), the Doctoral Politic Grant from Rennes 1 University.

\section{References}

[1] L. R. Baker and J. M. B. Webber. Thermoelastic Stress Analysis. Optica Acta: International Journal of Optics, 29(4):555-563, 1982.

[2] J.M. Dulieu-Barton and P. Stanley. Development and application of thermoelastic stress analysis. journal of strain analysis for engineering design. Journal of Strain Analysis for Engineering Design, 33:93-104, 1998.

[3] A. Chrysochoos. Energy-balance for elastic-plastic deformation at finite strain. J. Mec. Theo Appl., 4:589-614, 1985.

[4] A. Chrysochoos, O. Maisonneuve, G. Martin, H Caumon, and J. O. Chezeau. Plastic and dissipated work and stored energy. Nuclear Engineering and Design, 114:323-333, 1989.

[5] A. Chrysochoos and G. Martin. Tensile test microcalorimetry for thermomechanical behaviour law analysis. Mater. Sci. Eng. A, 108:25-32, 1989.

[6] P. Stanley and Wk Chan. Quantitative Stress-Analysis by Means of the Thermoelastic Effect. Journal of Strain Analysis for Engineering Design, 20:129-137, 1985. WOS:A1985APM1300001.

[7] Ak Wong, Sa Dunn, and Jg Sparrow. Residual-Stress Measurement by Means of the Thermoelastic Effect. Nature, 332:613-615, April 1988.

[8] F. A. Diaz, E. A. Patterson, R. A. Tomlinson, and J. R. Yates. Measuring stress intensity factors during fatigue crack growth using thermoelasticity. Fatigue \& Fracture of Engineering Materials \& Structures, 27:571-583, 2004.

[9] S. Barone and E. A. Patterson. Full-field separation of principal stresses by combined thermo- and photoelasticity. Experimental Mechanics, 36:318-324, 1996.

[10] D. Delpueyo, X. Balandraud, and M. Grédiac. Calorimetric signature of the Portevin-Le Chatelier effect in an aluminum alloy from infrared thermography measurements and heat source reconstruction. Materials Science and Engineering: $A$ - Structural materials properties microstructure and processing, 651:135 - 145, 2016. 
[11] J. R. Samaca Martinez, J.-B. Le Cam, X. Balandraud, E. Toussaint, and J. Caillard. Thermal and calorimetric effects accompanying the deformation of natural rubber. part 2: quantitative calorimetric analysis. Polymer, 54:2727 - 2736, 2013.

[12] B. Berthel, B. Wattrisse, A. Chrysochoos, and A. Galtier. Thermoelastic analysis of fatigue dissipation properties of steel sheets. Strain, 43:273-279, 2007.

[13] T. Boulanger, A. Chrysochoos, C. Mabru, and A. Galtier. Calorimetric analysis of dissipative and thermoelastic effects associated with the fatigue behavior of steels. International Journal of Fatigue, 26:221-229, 2007.

[14] A. Chrysochoos, C. Licht, and. Peyroux. Une modélisation thermomécanique unidimensionnelle de la propagation d'un front de changement de phase dans un monocristal d'AMF. Comptes Rendus Mécanique, 331:25-32, 2003.

[15] A. Chrysochoos. Thermomechanical Analysis of the Cyclic Behavior of Materials. Procedia IUTAM, 4:15-26, 2012.

[16] J. M. Dulieu-Barton, R. K. Fruehmann, and S. Quinn. A Full-field Stress Based Damage Assessment Approach for In-situ Inspection of Composite Structures. Key Engineering Materials, 569-570: 3-10. 2013.

[17] T. R. Emery and J. M. Dulieu-Barton. Thermoelastic Stress Analysis of damage mechanisms in composite materials. Compos. Pt. A-Appl. Sci. Manuf., 41:1729-1742, 2010.

[18] R. K. Fruehmann, W. Wang, J. M. Dulieu-Barton, and S. Quinn. The Application of Thermoelastic Stress Analysis to Evaluate Debond Damage in Composite Sandwich Structures. In Advances in Experimental Mechanics Viii, volume 70, pages 470-475. 2011.

[19] W. Wang, G. Martakos, J. M. Dulieu-Barton, J. H. Andreasen, and O. T. Thomsen. Fracture behaviour at tri-material junctions of crack stoppers in sandwich structures. Composite Structures, 133:818833,2015

[20] R. C. Waugh, J. M. Dulieu-Barton, and S. Quinn. Modelling and evaluation of pulsed and pulse phase thermography through application of composite and metallic case studies. Ndt \& $E$ International, 66:52-66, 2014.

[21] J. R. Samaca Martinez, J.-B. Le Cam, X. Balandraud, E. Toussaint, and J. Caillard. Thermal and calorimetric effects accompanying the deformation of natural rubber. part 1: Thermal characterization. Polymer, 54:2717 - 2726, 2013.

[22] R. Weichert and K. Schönert. Heat generation at the crack tip of a moving crack. Journal of Mechanics and Physics of Solids, 26:151-161, 1978.

[23] C. Pouvreau, M. Drissi-Habti, K. Michel, B. Bureau, J.-C. Sangleboeuf, C. Boussard-Pledel, T. Rouxel, and J.-L. Adam. Mechanical properties of a tas fiber: a preliminary study. Journal of Non-Crystalline Solids, 316(1):131 - 137, 2003.

[24] M.V Swain, J.C Metras, and C.G Guillemet. A deformation and fracture mechanics approach to the scoring and breaking of glass. Journal of Non-Crystalline Solids, 38-39, Part 1:445 - 450, 1980.

[25] J.-P. Guin and S. M. Wiederhorn. Crack growth threshold in soda lime silicate glass: role of hold-time. Journal of Non-Crystalline Solids, 316(1):12 - 20, 2003.

[26] J.-B. Le Cam, E. Robin, X. Balandraud, and E. Toussaint. A new experimental route in thermomechanics of inorganic glasses using infrared thermography. Journal of Non-Crystalline Solids, 366:6469, 2013. 
[27] E. Robin, J.-B. Le Cam, X. Balandraud, E. Toussaint, and L. Brilland. First steps towards the thermomechanical characterization of chalcogenide glass using quantitative infrared thermography. Journal of Non-Crystalline Solids, 391:101-105, 2014.

[28] G. Corvec, E. Robin, J.-B. Le Cam, J.-C. Sangleboeuf, and P. Lucas. Improving spatio-temporal resolution of infrared images to detect thermal activity of defect at the surface of inorganic glass. Infrared Physics \& Technology, 77:193-202, 2016.

[29] V. Chean, E. Robin, R. El Abdi, J-C Sangleboeuf, and P. Houizot. Use of the mark-tracking method for optical fiber characterization. Optics and Laser Technology, 43(7):1172-1178, 2011.

[30] Q.S. Nguyen, P. Germain, and P. Suquet. Continuum thermodynamics. J App/ Sci, 50:1010-1020, 1983.

[31] N. Sathon and J. M. Dulieu-Barton. Evaluation of sub-surface stresses using thermoelastic stress analysis. Appl. Mech. Mater, 7/8:153-158, 2007.

[32] A. Chrysochoos and H. Louche. An infrared image processing to analyse the calorific effects accompanying strain localisation. Int J Eng Sci, 38:1759-1788, 2000.

[33] S. Offermann, J. L. Beaudoin, C. Bissieux, and H. Frick. Thermoelastic stress analysis under nonadiabatic conditions. Experimental Mechanics, 37(4):409-413, 1997. 
Fig. 1. specimen geometry (dimensions in $\mathrm{mm}$ )

Fig. 2. overview of the experimental setup

Fig. 3. relative displacement of the white mark versus the image number once the preloading is applied

Fig. 4. $\mathrm{Y}$ versus $\mathrm{X}$ at the white mark \#1

Fig. 5. temperature evolution in the contact area (point A) 
Fig. 6. temperature variation map (a) full scale (b) reduced scale

Fig. 7. temperature variation (a) at points $A$ and $D(b)$ at points $B$ and $C$

Fig. 8. map of the signed $\Delta \operatorname{tr}(\sigma)$ (a)full scale (b) magnification of the contact zone in the boxed zone in Fig.8(a)

Fig. 9. solid finite element mesh

Fig. 10. experimental and numerical fields of hydrostatic pressure

Fig. 11. zoom in of the boxed zone in Fig. 10

Fig. 12. value of the hydrostatic pressure along a profile defined between the contact zones 
Fig. 13. heat source maps at image 285

Fig. 14. heat source variation (a) at points $A$ and $D(b)$ at points $B$ and $C$ 